\title{
Classification of X-ray solar flares regarding their effects on the lower ionosphere electron density profile
}

\author{
D. P. Grubor ${ }^{1}$, D. M. Šulić ${ }^{2}$ and V. Žigman ${ }^{3}$ \\ ${ }^{1}$ University of Belgrade, Belgrade, Faculty of Mining and Geology, Physics Cathedra, Djusina 7, 11000, Belgrade, Serbia \\ ${ }^{2}$ Institute of Physics, Belgrade, Pregrevica 118, Zemun, 11001, Belgrade, Serbia \\ ${ }^{3}$ University of Nova Gorica, Vipavska cesta 13, 5001 Nova Gorica, Slovenia
}

Received: 25 January 2007 - Revised: 12 February 2008 - Accepted: 24 April 2008 - Published: 18 June 2008

\begin{abstract}
The classification of X-ray solar flares is performed regarding their effects on the Very Low Frequency (VLF) wave propagation along the Earth-ionosphere waveguide. The changes in propagation are detected from an observed VLF signal phase and amplitude perturbations, taking place during X-ray solar flares. All flare effects chosen for the analysis are recorded by the Absolute Phase and Amplitude Logger (AbsPal), during the summer months of 20042007, on the single trace, Skelton (54.72 N, $2.88 \mathrm{~W}$ ) to Belgrade $(44.85 \mathrm{~N}, 20.38 \mathrm{E})$ with a distance along the Great Circle Path (GCP) $D \approx 2000 \mathrm{~km}$ in length.
\end{abstract}

The observed VLF amplitude and phase perturbations are simulated by the computer program Long-Wavelength Propagation Capability (LWPC), using Wait's model of the lower ionosphere, as determined by two parameters: the sharpness ( $\beta$ in $1 / \mathrm{km})$ and reflection height $\left(H^{\prime}\right.$ in $\left.\mathrm{km}\right)$. By varying the values of $\beta$ and $H^{\prime}$ so as to match the observed amplitude and phase perturbations, the variation of the D-region electron density height profile $N_{e}(z)$ was reconstructed, throughout flare duration. The procedure is illustrated as applied to a series of flares, from class $\mathrm{C}$ to $\mathrm{M} 5\left(5 \times 10^{-5} \mathrm{~W} / \mathrm{m}^{2}\right.$ at $0.1-$ $0.8 \mathrm{~nm}$ ), each giving rise to a different time development of signal perturbation.

The corresponding change in electron density from the unperturbed value at the unperturbed reflection height, i.e. $N_{e}(74 \mathrm{~km})=2.16 \times 10^{8} \mathrm{~m}^{-3}$ to the value induced by an M5 class flare, up to $N_{e}(74 \mathrm{~km})=4 \times 10^{10} \mathrm{~m}^{-3}$ is obtained. The $\beta$ parameter is found to range from $0.30-0.491 / \mathrm{km}$ and the reflection height $H^{\prime}$ to vary from $74-63 \mathrm{~km}$. The changes in $N_{e}(z)$ during the flares, within height range $z=60$ to $90 \mathrm{~km}$ are determined, as well.

Correspondence to: D. P. Grubor

(davorkag@rgf.bg.ac.yu)
Keywords. Ionosphere (Ionospheric disturbances) - Radio science (Ionospheric propagation) - Solar physics, astrophysics,and astronomy (Flares and mass ejections)

\section{Introduction}

The lower boundary of the D-region is known, as well as the upper edge of Earth-ionosphere waveguide, for very low frequency (VLF) wave propagation. VLF signals from transmitters, emitted with a well stabilized phase and amplitude, propagating under undisturbed ionospheric conditions, are continuously recorded by a number of world-wide receivers, showing the characteristic phase and amplitude diurnal variation. The most prominent change in the Earth-ionosphere waveguide is the day-to-night (and reverse) change. However, significant modifications of the propagating conditions happen due to severe changes in the lower ionosphere electron density, induced by solar X-ray flares. Although the main source of ionization, the Lyman- $\alpha$ emission, is enhanced during the flare event, the X-ray emission overwhelms its effect several times, leading to the increase in the D-region electron density by 1-2 orders of magnitude. Enhanced D-region density causes the change in the electrical conductivity at the upper waveguide edge along the trace of the VLF signal and consequently, gives rise to the change in all propagating parameters. These changes are clearly detected as the perturbation of phase and amplitude on the records of the diurnal VLF signal variation.

Although flare effects on the VLF signal are always well recognizable, they can vary substantially along different signal traces. The theory of VLF propagation through the Earthionosphere waveguide, at regular (quiet) ionospheric conditions, is well established (Wait, 1962). This theory predicts

Published by Copernicus Publications on behalf of the European Geosciences Union. 


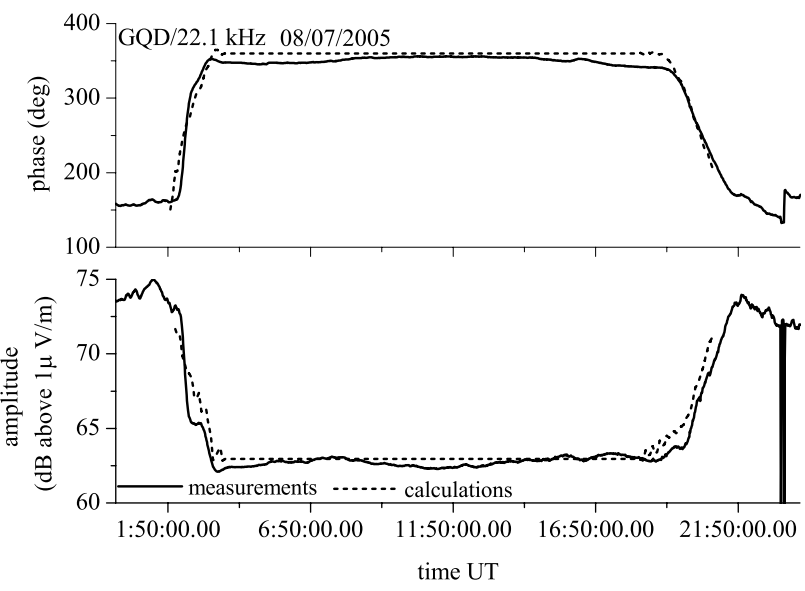

Fig. 1. Diurnal variations of the GQD signal phase (upper panel) and amplitude (lower panel), on 8 July 2005. AbsPAL recordings solid lines; LWPC simulation - short dashed lines.

the change in the VLF phase and amplitude along the particular transmitter to the receiver trace. Starting from this theory, a set of versatile computer programs for simulation of VLF propagation along any particular trace and at any regular diurnal, seasonal and solar cycle variations of the ionosphere has been developed. The set of programs has been continuously improved and one recent version, given by Ferguson (1998) and named Long Wavelength Propagation Capability (LWPC), is used in the present study.

The LWPC code consists of a set of programs which can be used separately according to the purpose of calculations. The default propagation model used by LWPC is the Long Wave Propagation Model (LWPM), which predicts an exponential increase in electrical conductivity within the ionospheric D-region. The height dependent conductivity parameter is given by $\omega_{r}(z)=\omega_{0}^{2} / \nu$, where $\omega_{0}$ is the electron plasma frequency, and $v$ is the effective electron-neutral collision frequency. The neutral particle density varies with height as $\exp (-\alpha z)$, where $1 / \alpha$ is the characteristic scale height. With the two parameters, $\beta$ and $H^{\prime}$, introduced by Wait and Spies (1964), the height profile of the conductivity parameter can be described in a simple way by:

$\omega_{r}(z)=2.5 \times 10^{5} \cdot \exp \left[\beta\left(z-H^{\prime}\right)\right]$,

where $\omega_{r}\left(H^{\prime}\right)=2.5 \times 10^{5} \mathrm{~s}^{-1}$. The parameter $\beta(1 / \mathrm{km})$, the so-called "sharpness" of the ionosphere lower boundary, gives the relative slope of the conductivity profile $\beta=\left(1 / \omega_{r}\right)\left(d \omega_{r} / d z\right)$. The parameter $H^{\prime}$ is the altitude at which the reflection of the VLF waves takes place. Using the $\left(\beta, H^{\prime}\right)$ pair, the electron density at a given altitude $z$ can be calculated, as it will be performed further in this paper. The ionospheric model is called "range exponential" with input data; the distance along the Great Circle Path (GCP) and its associated $\beta$ and $H^{\prime}$ parameters is used in the LWPC evaluations of VLF phase and amplitude.
While Wait's parameters, $\beta_{q}$ and $H_{q}^{\prime}$, describing the quiet diurnal, seasonal and solar cycle variations of the lower ionosphere are well determined, (Thomson, 1993; Thomson and Clilverd, 2000; McRae and Thomson, 2000), the values of these parameters describing the solar X-ray flare induced Dregion changes, are still under study. In recent papers, the $\beta$ and $H^{\prime}$ dependence on the solar X-ray flare peak irradiance has been deduced from VLF phase and amplitude observations (Thomson and Clilverd, 2001; McRae and Thomson, 2004; Thomson et al., 2004, 2005).

In the present study, VLF observations are used as well, but with the purpose to examine the development of phase and amplitude perturbation, as described through the values of $\beta$ and $H^{\prime}$, during the whole X-ray flare duration. The LWPC program has been run to obtain a pair of $\beta$ and $H^{\prime}$ parameters that leads to the best agreement between observed and calculated phase and amplitude perturbations, at characteristic stages of the particular flare development. Thus, from the time varying $\beta$ and $H^{\prime}$, the time variation of the electron density height profile during single flare event was reconstructed for the altitude range 60 to $90 \mathrm{~km}$. Conclusively, it was possible to attribute the specific pattern of the time-varying signal properties to the magnitude of peak $\mathrm{X}$ ray irradiance, for VLF-detected flare events.

\section{Recording, monitoring and simulation of VLF signal}

The observations reported presently were recorded by the VLF receiving system AbsPAL (Absolute Phase and Amplitude Logger - developed by the Radio and Space Physics Group of Otago University, New Zealand), which is situated at Belgrade (44.85 N, 20.38 E). The receiver has been in stable operation since 2004, providing simultaneous, wellcalibrated data (amplitude in $\mathrm{dB}$ above $1 \mu \mathrm{V} / \mathrm{m}$, and phase in deg.), from transmitters: NAA $/ 24.0 \mathrm{kHz}$, from Maine $(44.63 \mathrm{~N}, 67.28 \mathrm{~W}), \mathrm{NWC} / 19.8 \mathrm{kHz}$ from North-West Australia $(27.2 \mathrm{~N}, 114.98 \mathrm{E})$ and $\mathrm{GQD} / 22.1 \mathrm{kHz}$ from Skelton $(54.72 \mathrm{~N}, 2.88 \mathrm{~W})$. With the AbsPAL continuous operation, the received signals reveal regular, diurnal and seasonal amplitude and phase variations under unperturbed ionospheric conditions. The particularly stable daytime propagation on the GQD path is illustrated by the AbsPAL records on the quiet day of 8 July 2005, shown in Fig. 1; the phase and amplitude measurements (upper and lower plot, respectively) are represented by solid lines. The records are paralleled by calculations with the LWPC code "bearings", which operates with the specified input values $\beta_{q}=0.301 / \mathrm{km}$ and $H_{q}^{\prime}=74 \mathrm{~km}$, for the quiet daytime ionosphere (Ferguson, 1998). The effective radiated power from the GQD transmitter was estimated by the requirement that on the quiet day (i.e. 8 July 2005), the simulated amplitude and phase match the typical unperturbed phases and amplitudes measured at Belgrade. This procedure suggested that for the GQD transmitter (in the period under study, and at least in the direction to Belgrade), 
the value of $155 \mathrm{~kW}$ power is appropriate, and was, therefore, adopted in the present study. The corresponding results of the LWPC code simulation $\left(\beta_{q}=0.301 / \mathrm{km}, H_{q}^{\prime}=74 \mathrm{~km}\right)$ with $155 \mathrm{~kW}$ power are shown by dashed lines in Fig. 1. It is evident that measured and calculated phase and amplitude diurnal variation match very well. However, the LWPC default propagation model failed to reproduce the measured nighttime signal variation for any choice of the input parameters, when the particular GQD path is concerned. Therefore, only the well-simulated VLF signal, in the period between 02:00 and 21:00 UT, relevant for present study, is shown in Fig. 1.

When solar flares occur, sudden energy bursts in the X-ray domain appear most distinctly impressed on the VLF signal, by phase and amplitude with an abrupt increase or decrease, followed by the subsequent signal recovery within time intervals (typically less than an hour), which correspond to flare duration. Over 150 flare events, VLF-detected through May to August in the years 2004-2007, have been analyzed. Though apparently different depending on the path, the flareinduced VLF disturbances show stable patterns: while both phase and amplitude enhancement on long NAA and NWC paths are regularly detected, VLF perturbations on the short (1982 km in length) GQD path display more complexity, including both an decrease and increase, as well as oscillations, depending on the flare intensity (Grubor et al., 2005). Therefore, it is precisely the phase and amplitude perturbation of the GQD signal that we are concerned with in the present work. We relate the VLF signal perturbation measured by the AbsPAL to the solar X-ray irradiance, as monitored by the GOES 12 satellite; both data sets have 1-min resolution.

A representative example of flare-induced phase and amplitude perturbations, measured for the GQD signal, on the active day of 12 July 2005, in particular, is given in Fig. 2. The upper panel shows the solar X-ray irradiance as monitored by GOES 12, throughout the day (UT), indicating a sequence of flares of class $\mathrm{C}$ to $\mathrm{M}$, between sunrise and sunset.

The features of enhanced X-ray irradiance have a distinct impact upon the VLF phase and amplitude characteristic (solid lines on the middle and lower panel), which display different patterns peculiar to the GQD path, throughout a single active day. The measured unperturbed daytime values of phase and amplitude (short dashed lines on the middle and lower panel of Fig. 2), extracted from their diurnal variation, as presented in Fig. 1, are shown for reference. The correspondence between the flare event and the VLF signal perturbation is remarkable; moreover, the three mentioned types of phase/amplitude response to the flare are clearly present. Being registered on the same day and on a single path, they are expected to relate to the flare size, in particular.

In attempting to model and explain the variability of the GQD signal as received at Belgrade, two issues are important:

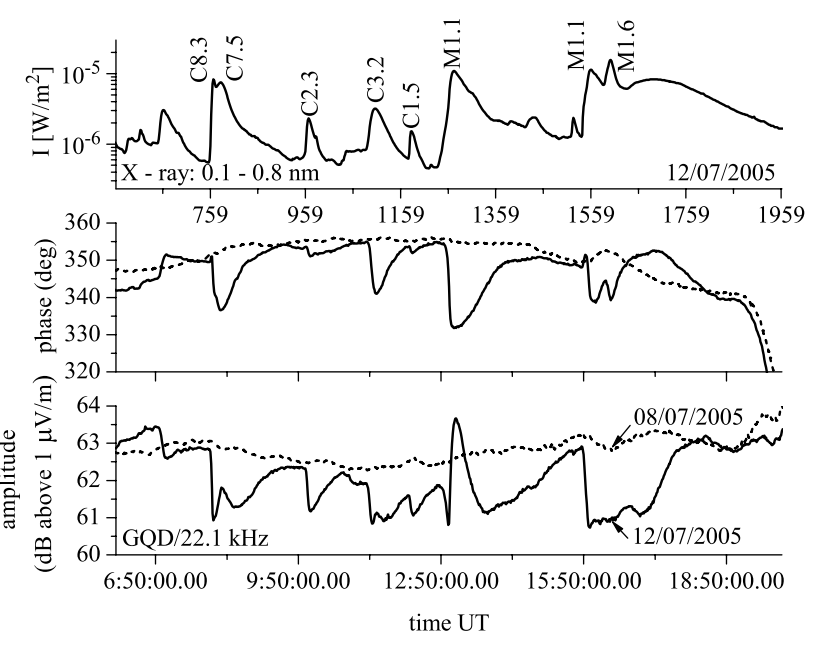

Fig. 2. Variation of X-ray irradiance and GQD signal phase and amplitude on 12 July 2005. X-ray irradiance (upper plot); phase (middle plot) and amplitude (lower plot) - solid lines. Measured phase and amplitude on quiet day (8 July 2005) - dashed lines.

1. The amplitude and phase of the GQD signal as recorded at the Belgrade site vary only slightly during quiet daytime (from local sunrise to local sunset), especially in the summer season (e.g. solid lines in Fig. 1). In other words, under regular conditions, stable daytime propagation allows the waveguide to be characterized by a single well-defined $\left(\beta_{q}=0.301 / \mathrm{km}, H_{q}^{\prime}=74.0 \mathrm{~km}\right)$ pair. This is readily adopted by the LWPC default propagation model, yielding phase and amplitude values shown by dashed lines in Fig. 1.

2. The GQD signal path covers less than two time zones, and is, for that reason, almost equally insolated at any particular moment, so that each segment of the path can be considered as equally affected by the flare. Consequently, it can be safely assumed that the variability of the GQD signal perturbations is strongly dominated by the variability of the X-ray flux itself: normal incidence peak irradiance and flare duration. These characteristics are given by the GOES $12 \mathrm{X}$-ray data lists in the wavelength range $0.1-0.8 \mathrm{~nm}$, which can be retrieved via the web site www.sec.noaa.gov.

\section{Determination of $\beta$ and $H^{\prime}$ under flare conditions}

The phase $\Delta P$, and amplitude $\Delta A$ perturbations are determined from the AbsPAL measurements on the quiet day $\left(P_{m q}, A_{m q}\right)$, and during the perturbation due to flare occurrence $\left(P_{m p}, A_{m p}\right)$ as:

$\Delta P=P_{m p}-P_{m q}$ and $\Delta A=A_{m p}-A_{m q}$.

These perturbations are then added to the simulated unperturbed (quiet) phase $P_{s q}$ and amplitude $A_{s q}$ values, given by 


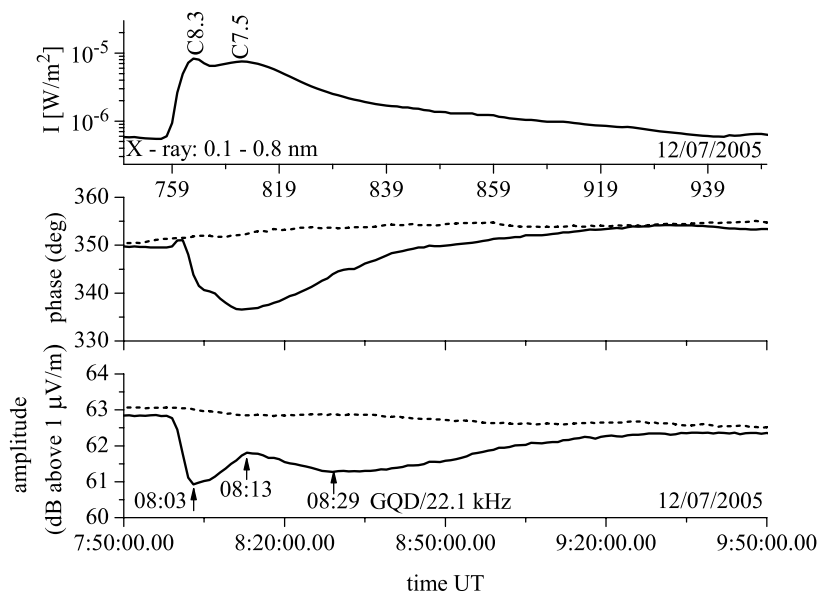

Fig. 3. Time variation of $\mathrm{X}$-ray irradiance, GQD signal phase and amplitude during C8.3 (08:03 UT) and C7.5 (08:12 UT) events on 12 July 2005 - solid lines. Extrema indicated by arrows and the corresponding UT. Phase and amplitude on the quiet day 8 July $2005-$ dashed lines.

the LWPC default propagation model at the receiver site, to obtain:

$P_{s p}=P_{s q}+\Delta P$ and $A_{s p}=A_{s q}+\Delta A$,

and the LWPC code is run to find the values of $\beta$ and $H^{\prime}$, which yield the particular perturbed phases and amplitudes $\left(P_{s p}, A_{s p}\right)$. This run of the LWPC program under perturbed ionospheric conditions is performed for several characteristic times during a single flare occurrence, to obtain $\beta$ and $H^{\prime}$ time variability. The unperturbed daytime values, $P_{s q}=359.86 \mathrm{deg}$ and $A_{s q}=62.95 \mathrm{~dB}$, obtained by the default propagation model with $\beta_{q}=0.301 / \mathrm{km}$ and $H_{q}^{\prime}=74 \mathrm{~km}$, are presently used for determining the perturbed simulated values, as given by Eq. (2).

Once $\beta$ and $H^{\prime}$ are estimated for a particular moment during the flare, the electron density profile for the lower ionosphere, corresponding to that moment, is obtained, according to the well-known model of exponential electron density increase with height (Wait, 1962; Thomson, 1993):

$N_{e}\left(z, H^{\prime}, \beta\right)=1.43 \times 10^{13} \exp \left(-0.15 H^{\prime}\right) \exp \left[(\beta-0.15)\left(z-H^{\prime}\right)\right]$.

In the present work, the electron density height profile is calculated for the interval from $z=60 \mathrm{~km}$ to $z=90 \mathrm{~km}$.

\section{Analysis of VLF measurements and calculations re- sults}

To illustrate the procedure described in Sect. 3, as well as the analysis of VLF data, we choose the series of flares that had occurred on 12 July 2005, and are presented, along with the VLF measurements in Fig. 2. The reason for this choice is: 1) the flare intensity (ranging from class $\mathrm{C}$ to $\mathrm{M}$ ) is characteristic for the majority of the flares that have been VLFrecorded at Belgrade in the years 2004-2007. (In this period, close to the minimum of the solar activity cycle, only a minor number of class- $X$ flares has been detected in the summer months and almost all of them out of local daytime).

2) All but one GQD-path characteristic type of flareinduced phase and amplitude perturbation are monitored on 12 July 2005, in response to particular flare events in the sequence occurring from sunrise to sunset. The flare-induced phase oscillation, which is not observed on 12 July 2005, is illustrated by the flare event monitored on 13 July 2004.

The characteristics of the flare-VLF event are given in Table 1: the first column identifies the flare by its class (maximum irradiance in $10^{-6} \mathrm{~W} / \mathrm{m}^{2}$ ) and time (UT) of the peak irradiance appearance. The second column lists the time at which the characteristic feature of the amplitude perturbation (minimum/maximum) is detected, followed by the measured phase and amplitude perturbation, and the LWPC-deduced $\beta$ and $H^{\prime}$ parameters that most accurately reproduce these perturbations. The corresponding electron density (3) at the reflection height of the quiet ionosphere $H_{q}^{\prime}=74 \mathrm{~km}$ is presented in the seventh column. For comparison, the results that follow from the solution of the electron continuity equation (called the " $N_{e}(t)$ method"), yielding the temporal evolution of electron density throughout the flare occurrence (Žigman et al., 2007), are listed as well. The flare-VLF events on 12 July 2005 are listed in the order of appearance. Two events on 13 July 2004 conclude Table 1.

Flare-VLF events from Table 1, that display three characteristic types of perturbation behaviour on the GQD path, are also presented graphically. Figures 3, 4, 5, which are "close ups" of Fig. 2, and Fig. 6 (for 13 July 2004), represent the simultaneous variations of the X-ray irradiance (upper panel), the measured perturbed (solid line) and quiet (dashed line) phase and amplitude (middle and lower panel, respectively), in function of UT. The extrema are indicated by arrows and the corresponding UT time of their occurrence, for which the LWPC code is run.

The first flare in the series on 12 July 2005 , of C8.3 class with peak irradiance $I=8.33 \times 10^{-6} \mathrm{~W} / \mathrm{m}^{2}$ at $08: 03 \mathrm{UT}$, induces the drop in phase and amplitude (with minimum at 08:03 UT), but before irradiance descends to the base level, a new, lower-intensity flare apparently occurs. The convolution of the irradiance time distributions of both flares results in a new peak at 08:12 UT, with irradiance $I=7.51 \times 10^{-6} \mathrm{~W} / \mathrm{m}^{2}$. We assume that further phase and amplitude perturbation is controlled by the C7.5 class flare. This causes a phase decrease to a minimum, but an oscillation in amplitude, with a maximum (08:13 UT) and the subsequent shallow minimum (08:29 UT), after which the amplitude recovers to its unperturbed value (Fig. 3). The duration of the flare event is about two hours.

The next occurring (Fig. 2) class C2.3 flare event with peak irradiance $I=2.31 \times 10^{-6} \mathrm{~W} / \mathrm{m}^{2}$ at 10:03 UT causes only 
Table 1. Measured amplitude and phase perturbations, deduced $\beta$ and $H^{\prime}$ and electron densities $N_{e}$, at different stages of flare occurrence: present study* and Žigman et al. (2007)**.

\begin{tabular}{|c|c|c|c|c|c|c|c|}
\hline \multirow{2}{*}{$\begin{array}{l}\text { flare class; } \\
\text { peak time UT }\end{array}$} & \multirow{2}{*}{ time UT } & \multirow{2}{*}{$\begin{array}{c}\Delta A \\
(\mathrm{~dB})\end{array}$} & \multirow{2}{*}{$\begin{array}{c}\Delta P \\
(\mathrm{deg})\end{array}$} & \multirow{2}{*}{$\beta\left(\mathrm{km}^{-1}\right)$} & \multirow{2}{*}{$H^{\prime}(\mathrm{km})$} & \multicolumn{2}{|c|}{$N_{e}(74 \mathrm{~km})\left(\mathrm{m}^{-3}\right)$} \\
\hline & & & & & & $*$ & $* *$ \\
\hline \multicolumn{8}{|c|}{12 July 2005} \\
\hline \multirow{3}{*}{ C7.5; 08:12 } & 08:03 & -2.09 & -8.07 & 0.410 & 68.4 & $2.15 \times 10^{9}$ & $2.67 \times 10^{9}$ \\
\hline & 08:13 & -1.04 & -15.66 & 0.400 & 67.0 & $4.09 \times 10^{9}$ & $2.92 \times 10^{9}$ \\
\hline & 08:29 & -1.62 & -9.73 & 0.395 & 68.5 & $1.90 \times 10^{9}$ & $1.78 \times 10^{9}$ \\
\hline $\mathrm{C} 2.3 ; 10: 03$ & 10:05 & -1.31 & -4.25 & 0.355 & 70.0 & $8.94 \times 10^{8}$ & $1.17 \times 10^{9}$ \\
\hline \multirow{3}{*}{ C $3.2 ; 11: 27$} & $11: 23$ & -1.47 & -9.49 & 0.360 & 69.0 & $1.31 \times 10^{9}$ & $1.90 \times 10^{9}$ \\
\hline & $11: 29$ & -1.19 & -14.44 & 0.415 & 67.4 & $3.34 \times 10^{9}$ & $2.71 \times 10^{9}$ \\
\hline & $11: 38$ & -1.46 & -11.12 & 0.385 & 68.3 & $1.94 \times 10^{9}$ & $2.33 \times 10^{9}$ \\
\hline C1.5; $12: 13$ & 1215 & -1.51 & -4.09 & 0.350 & 70.1 & $8.46 \times 10^{8}$ & $7.35 \times 10^{8}$ \\
\hline \multirow{3}{*}{ M1.1; 13:06 } & $12: 59$ & -1.69 & -8.18 & 0.374 & 69.0 & $1.40 \times 10^{9}$ & $3.10 \times 10^{9}$ \\
\hline & $13: 09$ & +1.07 & -23.07 & 0.490 & 64.8 & $1.96 \times 10^{10}$ & $9.16 \times 10^{9}$ \\
\hline & $13: 49$ & -1.68 & -10.95 & 0.390 & 68.3 & $2.0 \times 10^{9}$ & $4.92 \times 10^{9}$ \\
\hline \multirow{3}{*}{$\begin{array}{l}\text { M1.1; 15:59 } \\
\text { M1.6; 16:24 }\end{array}$} & $15: 58$ & -2.46 & -9.15 & 0.435 & 68.0 & $2.94 \times 10^{9}$ & $3.53 \times 10^{9}$ \\
\hline & $16: 03$ & -2.14 & -12.39 & 0.440 & 67.6 & $3.61 \times 10^{9}$ & $4.42 \times 10^{9}$ \\
\hline & $16: 18$ & -1.92 & -8.01 & 0.395 & 68.7 & $1.75 \times 10^{9}$ & $1.67 \times 10^{9}$ \\
\hline \multicolumn{8}{|c|}{13 July 2004} \\
\hline \multirow{3}{*}{ M5.4; 08:48 } & $08: 47$ & +2.02 & -15.23 & 0.430 & 65.0 & $1.04 \times 10^{10}$ & $1.52 \times 10^{10}$ \\
\hline & $08: 52$ & +4.65 & -7.09 & 0.475 & 63.0 & $4.02 \times 10^{10}$ & $4.35 \times 10^{10}$ \\
\hline & 09:12 & +1.05 & -10.27 & 0.360 & 67.0 & $2.69 \times 10^{9}$ & $1.84 \times 10^{10}$ \\
\hline \multirow{3}{*}{ M3; 12:08 } & $12: 07$ & +2.06 & -17.09 & 0.44 & 65.0 & $1,13 \times 10^{10}$ & $7.97 \times 10^{9}$ \\
\hline & 12:09 & $+3,46$ & $-15,65$ & 0.47 & 64.0 & $2.38 \times 10^{10}$ & $1.12 \times 10^{10}$ \\
\hline & $12: 53$ & -1.19 & -7.40 & 0.34 & 69.5 & $9,98 \times 10^{8}$ & $1.69 \times 10^{9}$ \\
\hline
\end{tabular}

a single minimum in the perturbation of the VLF signal, both in phase and in amplitude, as shown in the close up in Fig. 4.

The perturbations caused by two subsequent flares, the first with peak irradiance $I=3.18 \times 10^{-6} \mathrm{~W} / \mathrm{m}^{2}(\mathrm{C} 3.2)$ at 11:27 UT and the successive one with peak irradiance $1.52 \times 10^{-6} \mathrm{~W} / \mathrm{m}^{2}(\mathrm{C} 1.5)$ at $12: 13 \mathrm{UT}$ follow the same patterns as those caused by flares $\mathrm{C} 7.5$ and $\mathrm{C} 2.3$, respectively, as can be readily seen from Fig. 2 . In particular, the $\mathrm{C} 1.5$ class is the shortest event in the series, lasting around $40 \mathrm{~min}$.

The M1.1 class flare $\left(I=1.1 \times 10^{-5} \mathrm{~W} / \mathrm{m}^{2}\right)$ at 13:06 UT, was the one with the longest duration on 12 July 2005, extended in the descending branch, probably due to some superimposed $\mathrm{C}$ class flares. According to VLF measurements, the ionosphere was perturbed for almost three hours. The type of phase perturbation has the same pattern as for the flare C7.5, but the intermediate peak in amplitude oscillation is much more pronounced and exceeds the quiet amplitude level (Fig. 5). The characteristic features of the amplitude perturbation are identified as 1) an abrupt decrease to minimum (12:58 UT) preceding the peak X-ray irradiance, 2) amplitude maximum reached at 13:09 UT with a time delay of 3 min with respect to peak X-ray irradiance, and 3) passage through a minimum (13:49 UT) during the long-lasting amplitude depression.

A double peaked flare (or two convoluted $\mathrm{M}$ class flares), with the first peak at 15:59 UT, (Fig. 2), caused the amplitude drop to last more than two hours. In the time interval between the two X-ray peaks, the recovery of the phase occurs, but there is no significant recovery of amplitude. The magnitude of the flare effect on the VLF signal can be clearly seen from Fig. 2. 


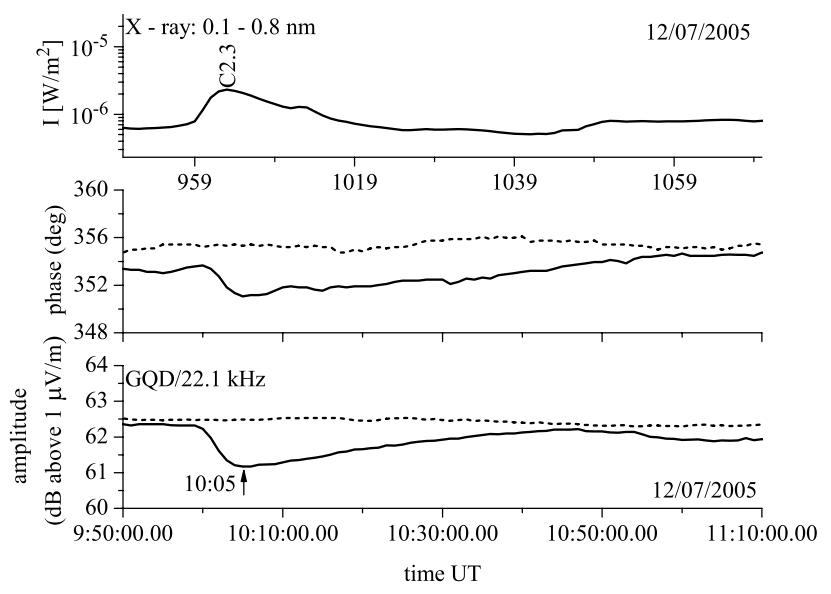

Fig. 4. Time variation of $X$-ray irradiance, GQD signal phase and amplitude during C2.3 (10:03) event on 12 July 2005 - solid lines. Extrema indicated by arrows and the corresponding UT. Phase and amplitude on the quiet day 8 July 2005 - dashed lines.

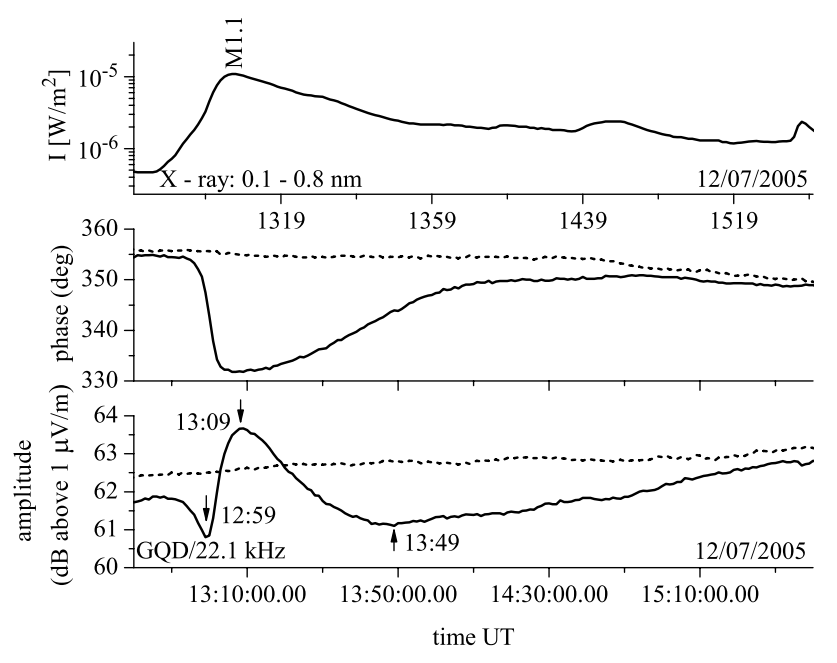

Fig. 5. Time variation of $X$-ray irradiance, GQD signal phase and amplitude during M1.1 (13:06 UT) event on 12 July 2005 solid lines. Extrema indicated by arrows and the corresponding UT. Phase and amplitude on the quiet day 8 July 2005 - dashed lines.

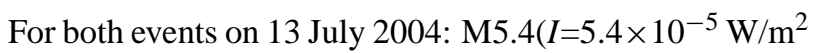
at $08: 48)$, and $\mathrm{M} 3.0\left(I=3.0 \times 10^{-5} \mathrm{~W} / \mathrm{m}^{2}\right.$ at $\left.12: 08\right)$, the main feature of the amplitude perturbation is a pronounced peak exceeding for several $\mathrm{dBs}$ the pre-flare amplitude value (Fig. 6). The peak is preceded by a short lasting drop in amplitude, followed by a depression with amplitude minimum appearing $50 \mathrm{~min}$ and $45 \mathrm{~min}$ after the M5.4 and M3.0 peak X-ray irradiance, respectively. Contrary to previous examples (with a single minimum in phase perturbation), the phase goes through an oscillation. The appearance of an oscillation in the phase (as well as in the amplitude) is apparently peculiar to larger flares, as illustrated by the M-class

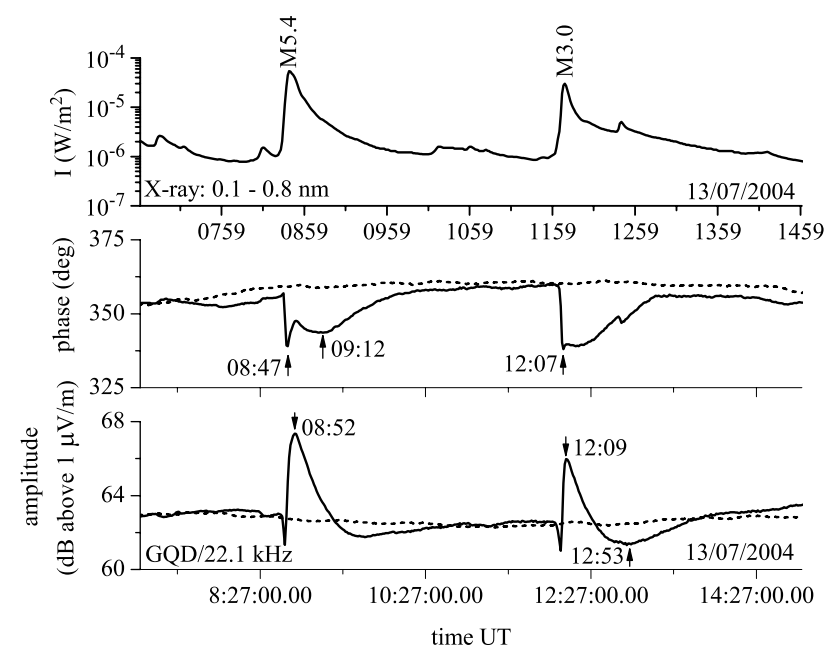

Fig. 6. Simultaneous variation of X-ray irradiance and GQD signal phase and amplitude during M5.4 (08:48 UT) and M3.0 (12:08 UT) events on 13 July 2004 - solid lines. Extrema indicated by arrows and the corresponding UT. Phase and amplitude on the quiet day 8 July 2005 - dashed lines.

flares on 13 July 2004. Both flares occur at pre-flare ionospheric conditions similar to the regular ones on 8 July 2005. The additional perturbation in the phase recovery, after the M3.0 flare, is due to the C5-class flare (12:49UT) occurrence.

\section{Discussion}

The guidelines for estimation of $\beta$ and $H^{\prime}$ parameters at perturbed ionospheric conditions are given by Thomson and Clilverd (2001) and McRae and Thomson (2004). In these papers the dependences of $\beta$ and $H^{\prime}$ parameters on the peak $\mathrm{X}$-ray flare irradiance were presented. However, the short GQD signal path reveals characteristics different than those of the traces treated in these papers (mainly long over sea paths) and therefore, requires an independent trial and error approach for $\beta$ and $H^{\prime}$ estimation. The $\beta$ and $H^{\prime}$ parameters determined by the simulation procedure described in Sect. 3, at characteristic stages of amplitude perturbation development (Table 1), are presented as a function of the X-ray flare irradiance on Figs. 7 and 8, respectively.

We compare our results (circles in Figs. 7 and 8) with the ones reported by Thomson et al. (2005), and from the previous extensive study of McRae and Thomson (2004), who have compiled and critically analyzed flare VLF data from four transmitters in the range $10.2-24.8 \mathrm{kHz}$ (squares in Figs. 7 and 8). As the latter data are deduced from measurements on significantly larger (of the order of several Mm) and mainly over sea paths, the agreement can be considered as fairly good. 


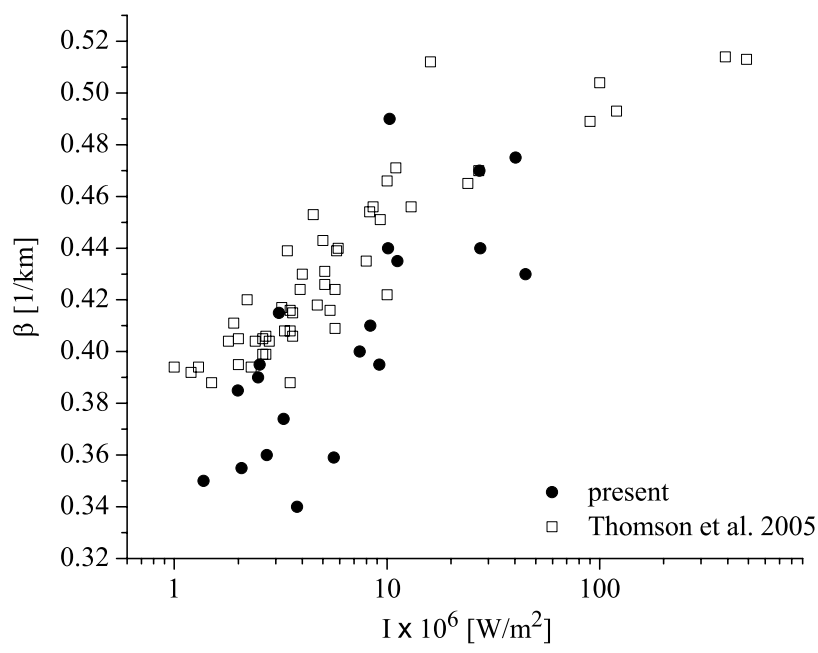

Fig. 7. Ionospheric sharpness, $\beta$ versus $\mathrm{X}$-ray irradiance. Present results: circles; Thomson et al. (2005): squares.

The presently evaluated $\beta$ and $H^{\prime}$ parameters display the same tendency - an increase in $\beta$ and a decrease in $H^{\prime}$, with increasing X-ray flare irradiance $I\left(\mathrm{~W} / \mathrm{m}^{2}\right)$, as do the results of Thomson et al. (2005) and McRae and Thomson (2004). To some extent, the spread of the present values of $\beta$ is due to the consideration of different stages of a single flare. If only the flare peak values were taken into account, as in the above-cited papers, the increasing trend would be more apparent. Additionally, the $P_{s p}$ and $A_{s p}$ values are found to be noticeably more sensitive to the variation in $H^{\prime}$ than to the variation in $\beta$. This contributes, at least partly, to both the spread of the $\beta$ values, on the one hand, and to the coherence of the $H^{\prime}$ values, on the other.

The electron density (Eq. 3) at quiet reflection height $z=H_{q}^{\prime}=74 \mathrm{~km}$, for the presently obtained $\left(\beta, H^{\prime}\right)$ pairs versus X-ray flare irradiance, is shown by squares in Fig. 9. An overall good agreement is seen with the results according to the $N_{e}(t)$ method of Žigman et al. (2007), applied to the flare-VLF events listed in Table 1 (circles in Fig. 9); both sets of $N_{e}$ data display the well-known increasing trend with increasing X-ray irradiance (Mitra, 1974, and references therein).

The redistribution of ionization at the upper waveguide boundary due to flare occurrence is illustrated by the electron density height profile changes during the $\mathrm{C} 7.5$ class flare on 12 July 2005, as shown in Fig. 10. At unperturbed (quiet) ionospheric conditions (short dashed line), there is a moderate increase in electron density from $10^{7} \mathrm{~m}^{-3}$ at $60 \mathrm{~km}$ height, to about $10^{9} \mathrm{~m}^{-3}$ at the upper boundary of the Dlayer $(90 \mathrm{~km}$ height). The abrupt rise in X-ray irradiance at 08:03 UT causes the lowering of the altitude at which the electron density reaches $10^{9} \mathrm{~m}^{-3}$, for about $13 \mathrm{~km}$, (dashed line). Though the three pertaining values of $\beta$ (Table 1) differ only by at most $4 \%$, the slope of the (dashed) line at 08:03

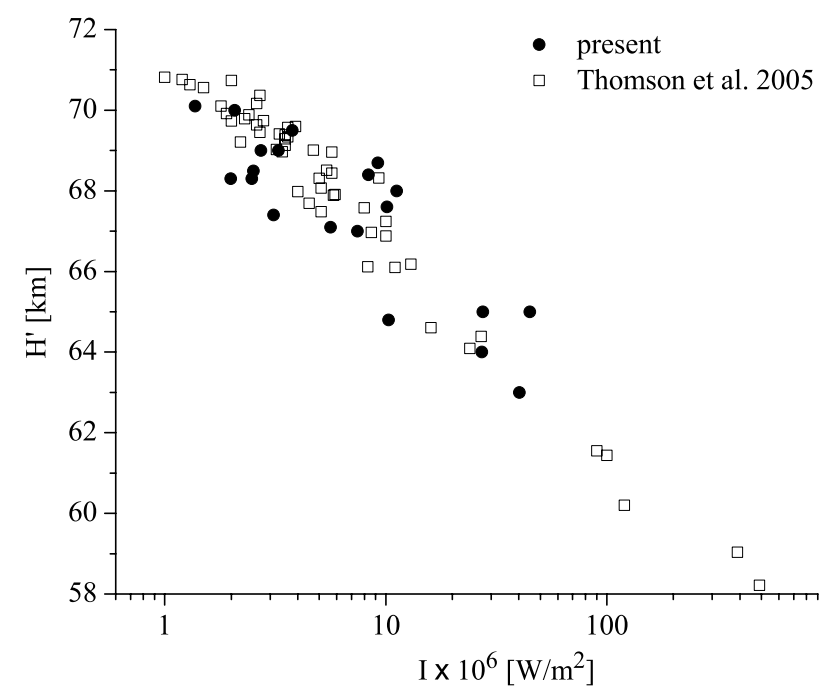

Fig. 8. Reflection height, $H^{\prime}$ versus X-ray irradiance. Present results: circles; Thomson et al. (2005): squares.

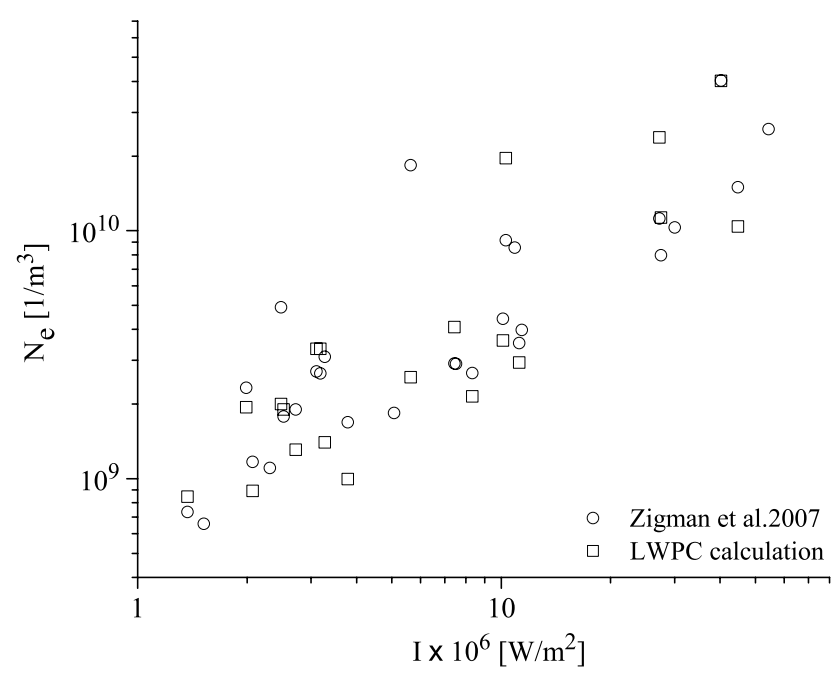

Fig. 9. Electron density versus $X$-ray flare irradiance, at $74 \mathrm{~km}$ height: present results squares - from $\beta$ and $H^{\prime}$ parameters estimated for the flare events on 12 July 2005 and 13 July 2004; circles $-N_{e}(t)$ method (Žigman et al., 2007) applied to the same series of flares.

(as well as the value of $\beta$ ) is the largest. With the time delay (Mitra, 1974; Žigman et al., 2007) of one minute after the X-ray peak irradiance, namely at 08:13 UT, the intermediate peak in amplitude appears. The corresponding electron density profile moves to even higher electron densities (solid line), with slightly lower slope as compared to the the density profile at 08:03 UT. For the amplitude passing through the second minimum at 08:29, the slope of the electron density profile (dotted line) is decreased further, towards the slope of the regular ionosphere electron density (short dashed line), revealing the beginning of the ionosphere recovery period. 


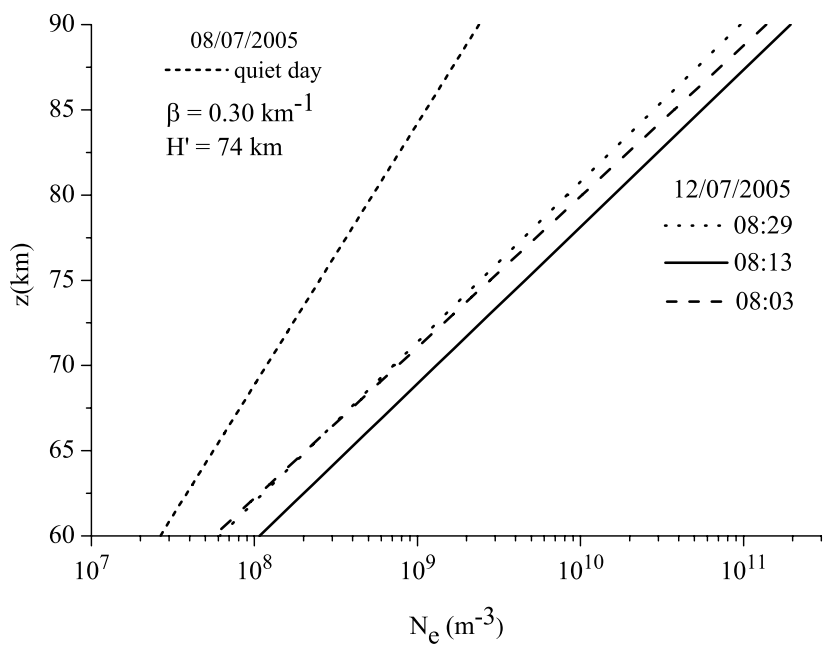

Fig. 10. Changes in the electron density profile in the course of flare C7.5. Pertaining phase and amplitude variation, as shown in Fig. 3.

These changes in electron density profile are of crucial importance for the Earth-ionosphere VLF propagation path. The LWPC program, with the incorporated range exponential model, defined by properly chosen $\beta$ and $H^{\prime}$ parameters, enables the simulation of VLF amplitude and phase variation along the propagation path, at any moment during the flare. The amplitude and phase variation along the GCP distance from the GQD transmitter to Belgrade receiver, calculated for characteristic times/stages during the $\mathrm{C} 7.5$ class flare on 12 July 2005, is given in Fig. 11.

The modal interference minima (e.g. Wait, 1962, Chapter IX, V.L.F. Propagation - theory and experiment), of the amplitude and phase variation along the GCP path, at regular conditions are clearly displayed on the typical quiet day of 8 July 2005 (short-dashed line in Fig. 11). The main modal minimum appears at $750 \mathrm{~km}$ from the transmitter, indicating the place on the GCP most sensitive to the VLF-monitoring of solar flares. On 12 July 2005, at the time of the C7.5 class flare occurrence, all modal minima are seen to move towards the transmitter (Thomson and Clilverd, 2001). The typical displacements of the modal minima are strongly dependent on the lowering of the reflection height: thus the reflection heights at 08:03 UT and at 08:29 UT, which differ by only $0.1 \mathrm{~km}$, result in the overlapping of the pre-flare (dashed) and post-flare (dotted) curves on the plots in Fig. 11. The amplitude variation during the flare indicates the tendency of formation of the modal minimum in the vicinity of the receiver (lower inset). It is clearly seen that the LWPC simulated phase and amplitude, at the receiver, are decreased with respect to the quiet day value at all stages of the flare, in accordance with the measurements, presented in Fig. 3. This agreement between the simulated amplitude and phase along the GCP at the particular receiver site, and AbsPAL measurements holds for all the flare events analyzed.

\section{Conclusion}

VLF phase and amplitude of the GQD/22.1 kHz transmitter recorded by the Belgrade AbsPAL have been analyzed for the survey of 150 solar X-ray flare events, occurring in the period May to August 2004-2007. The measured phase and amplitude perturbations and their characteristic features, specific for the short GQD path, which is mostly over land, have been related to the solar flare intensities, as monitored by the GOES-12 satellite, the representative examples being given in Figs. 4 to 6.

The simulation of the VLF propagation at quiet daytime ionospheric conditions, with the well-established LWPC code, have been confirmed by phase and amplitude measurements performed at Belgrade. On the basis of this agreement, we have applied the LWPC algorithm to different temporal stages of the measured phase and amplitude perturbation, within a single flare event. Thus, a particular $\left(\beta, H^{\prime}\right)$ pair is assigned to each phase/amplitude perturbation feature induced by the flare considered (Table 1).

Good agreement is found between the $\left(\beta, H^{\prime}\right)$ values derived presently and the ones analyzed and compiled by McRae and Thomson (2004) and presented also in Thomson et al. (2005), though the latter are deduced for primarily long, over-sea paths, with one $\left(\beta, H^{\prime}\right)$ pair assigned to each flare maximum. However, we have taken into account that $\beta$ (and $H^{\prime}$ as well) change during solar flares: $\beta$ increasing $\left(H^{\prime}\right.$ decreasing) in the ascending branch of the flare, and quite the contrary in the descending branch towards irradiance relaxation. Thus we have treated the $\left(\beta, H^{\prime}\right)$ pair as functions of time throughout the duration of the flare.

In this way, from the model that basically rests on the height profile (Eq. 3), a time resolved electron density profile is obtained (e.g. Fig. 10), which compares favorably with the prediction of the independent $N_{e}(t)$ model. In spite of the complexity of the phase/amplitude behaviour on the GQD path, the increase in the electron density with increasing flare intensity is remarkably confirmed (Fig. 9). The results arrived at allow for the classification of solar X-ray flares by their effect on the lower ionosphere and indicate the possible mechanisms of VLF propagation in perturbed conditions:

1. Low C-class flares cause a small decrease of amplitude and phase up to a single minimum appearing with a time delay of a few minutes after peak X-ray irradiance (flare events C2.3 and C1.5). A likely explanation for the decrease in amplitude can be found in the field strength attenuation as the signal penetrates the D-region undergoing ionization redistribution. Along with the electron density increase, the upper boundary of the waveguide drifts slightly downwards to lower heights. In consequence, the modal minimum is formed close to the receiver, additionally decreasing the amplitude of the received signal. 


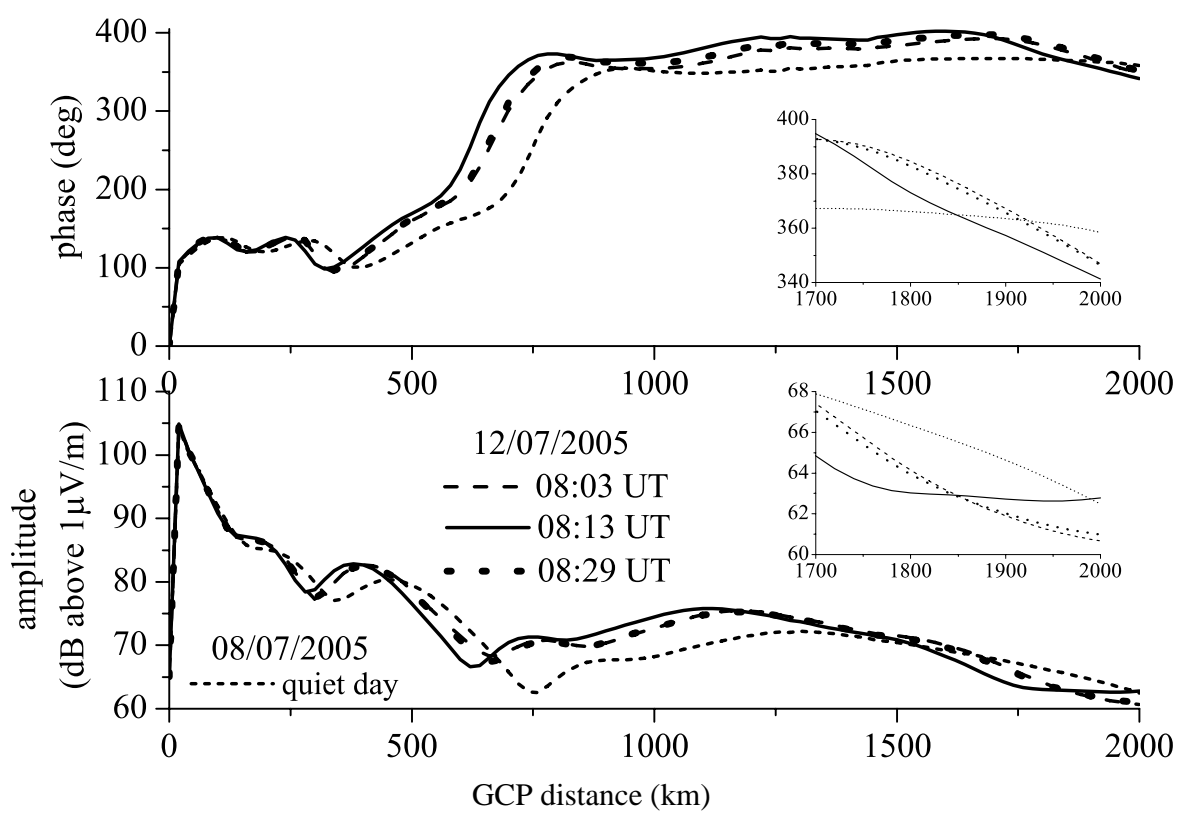

Fig. 11. Variation of phase and amplitude of the GQD signal along the GCP distance from Skelton to Belgrade, calculated for three characteristic times during the C7.5 event on 12 July 2005 (see Fig. 3). The close up of the last $300 \mathrm{~km}$ on GCP is added to the phase and amplitude plot.

2. C-class flares above $\mathrm{C} 3$ cause a further increase in the sharpness at the waveguide upper boundary and a decrease in the reflection height. Preceded and followed by minima (e.g. Fig. 3), the intermediate amplitude maximum appears, but does not exceed the preflare amplitude value, suggesting the transient stage to the mirror-like wave reflection. The phase shows only one minimum, approximately coinciding with the amplitude intermediate peak. The effect of C7.5 and C3.2 events on 12 July 2005 , represent this type of perturbations.

3. The increase in the flare irradiance above the M-class level sets the waveguide propagation conditions characterized by high sharpness and very low reflection height. The intermediate maximum exceeds the pre-flare amplitude value, indicating that a mirror-type reflection takes place. The descent of the reflection height for almost $10 \mathrm{~km}$ reduces the phase on the effectively shortened path, advancing the phase at the receiver. The effect of the M1.1 event (Fig. 5) represents this type of perturbation $\left(\beta=0.49, H^{\prime}=64.8\right.$, Table 1$)$.

4. The prominent intermediate amplitude peaks, well above the amplitude preflare value, as seen for events M5.4 and M3.0, indicate a mirror-type reflection at very low reflection heights, $63 \mathrm{~km}$ and $64 \mathrm{~km}$, respectively. Unlike the phase variation during the C7.5- and M1.1class events, i.e. with a single minimum, the intermediate peak in phase appears. The phase peak is due to the slight displacement of phase modal minima toward the transmitter. The tiny phase peak, present in the case of the M3.0 event, suggests that flares of higher than M3.0 class have to take place, to give rise to the oscillation type of phase perturbation.

With $\beta$ and $H^{\prime}$ taken as $\beta(I(t))$, and $H^{\prime}(I(t))$ an insight is gained into the VLF propagation along the GCP (displacements of modal minima) and the redistribution of the electron density height profile during the ascending and descending stages of the flare evolution (Fig. 11). The correspondence of $N_{e}(z)_{t}$ at the specified time, according to LWPC and of $N_{e}(t)_{h}$ for a specified height, according to the $N_{e}(t)$ model, anticipate the complementary way in which the two approaches may be merged into the unified description, providing a more realistic $N_{e}(t, z)$ electron density profile under flare conditions.

Acknowledgements. The supports of the Ministry of Science and Environmental Protection of Serbia, project 141044 (D. P. Grubor and D. M. Šulić) and the Slovenian Research Agency, contract P2-0056 (V. Žigman) are gratefully acknowledged. The research was performed as part of the bilateral Slovenian-Serbian project BI-SCG05/06. The theme was a research topic within the COST Action 724 in the years 2004-2007. The authors thank N.R. Thomson, C.J. Rodger for supplying the $\beta$ and $H^{\prime}$ results (Thomson et al., 2005; McRae and Thomson, 2004), in tabular form.

Topical Editor U.-P. Hoppe thanks two anonymous referees for their help in evaluating this paper. 


\section{References}

Ferguson, A. J.: Computer Programs for Assessment of LongWavelength Radio Communications, Version 2.0, Technical document 3030, Space and Naval Warfare Systems Center, San Diego CA 92152-5001, 1998.

Grubor, D., Šulić D., and Žigman, V.: Influence of solar X-ray flares on the Earth-ionosphere waveguide, Serb. Astron. J., 171, 29-35, 2005.

McRae, M. W. and Thomson, N. R.: VLF phase and amplitude: daytime ionospheric parameters, J. Atmos. Sol.-Terr. Phys., 62, 609-618, 2000.

McRae, M. W. and Thomson, N. R.: Solar flare induced ionospheric D-region enhancements from VLF phase and amplitude observations, J. Atmos. Sol.-Terr. Phys., 66, 77-87, 2004.

Mitra, A. P.: Ionospheric Efects of Solar Flares, Astrophysics and space science library, 46, D. Reidel Publishing Company, Boston, 1974.

Thomson, N. R.: Experimental daytime VLF ionospheric parameters, J. Atmos. Sol.-Terr. Phys., 55(2), 173-184, 1993.
Thomson, N. R. and Clilverd, A. M.: Solar cycle changes in daytime VLF subionospheric attenuation, J. Atmos. Sol.-Terr. Phys., 62, 601-608, 2000.

Thomson, N. R. and Clilverd, A. M.: Solar flare induced ionospheric D-region enhancements from VLF amplitude observations, J. Atmos. Sol.-Terr. Phys., 63, 1729-1737, 2001.

Thomson, N. R., Rodger, J. C., and Dowden, L. R.: Ionosphere gives size of greatest solar flare, Geophys. Res. Lett., 31, L06803, doi:10.1029/2003GL019345, 2004.

Thomson, N. R., Rodger, J. C., and Clilverd, A. M.: Large solar flares and their ionospheric D-region enhancements, J. Geophys. Res., 110, A06306, doi:10.1029/2005JA011008, 2005.

Wait, R. J.: Electromagnetic waves in stratified media, Pergamon Press, 1962.

Wait, R. J. and Spies, P. K.: Characteristics of the Earth-ionosphere waveguide for VLF radio waves, Technical Note 300, National Bureau of Standards, U.S. Department of Commerce, 1964.

Žigman, V., Grubor, D., and Šulić, D.: D-region electron density evaluated from VLF amplitude time delay during X-ray solar flares, J. Atmos. Sol.-Terr. Phys., 69(7), 775-792, 2007. 